\title{
Briófitos en los troncos de árboles como indicadores de la alteración en bosques montanos de Panamá
}

\author{
Georgina Guerra ${ }^{1}$, Clotilde Arrocha ${ }^{1}$, Gloria Rodríguez ${ }^{1}$, Jorge Déleg ${ }^{2}$ \& Ángel Benítez ${ }^{3 *}$ \\ 1. Herbario Universidad Autónoma de Chiriquí (UCH). Chiriquí, Panamá; ginnasj@hotmail.com, \\ clotilde.arrocha1@unachi.ac.pa, gloriakatiuska23@hotmail.es \\ 2. Maestría en Biología de la Conservación y Ecología Tropical, Universidad Técnica Particular de Loja, San Cayetano \\ s/n, Loja 1101608, Ecuador; jadeleg@utpl.edu.ec \\ 3. Departamento de Ciencias Biológicas, Universidad Técnica Particular de Loja, San Cayetano Alto s/n, Loja 1101608, \\ Ecuador; arbenitez@utpl.edu.ec \\ * Correspondencia
}

Recibido 11-IX-2019. Corregido 20-XII-2019. Aceptado 09-III-2020.

\begin{abstract}
Bryophytes on tree trunks as indicators of montane forest disturbance in Panama. Introduction: Tropical montane forests are characterized by high species richness and endemism of bryophytes. However, this diversity is highly sensitive to microclimatic changes related to anthropogenic disturbance. Objective: In this study, we assessed the richness and composition of epiphytic bryophytes on the trunks of trees in undisturbed and disturbed montane forests on the Western slope of the Baru Volcano National Park. Methods: Undisturbed forests were highly humid, while disturbed forests showed high light levels and temperature. In each tree we measured the presence and cover of epiphytic bryophytes in 160 quadrants of $20 \times 30 \mathrm{~cm}$. Results: In total, we recorded 86 species (49 liverworts and 37 mosses). Bryophytes richness was positively influenced by disturbance, with disturbed forests showing a high number of sun epiphytes. Bryophyte community composition was different in each forest type, with shade epiphytes confined to primary forests and sun epiphytes restricted to disturbed sites. Conclusions: Bryophyte communities were effective indicators of montane forests disturbance on the Baru Volcano. Thus, protection of the undisturbed forests remnants might help to preserve a rich and diverse community of epiphytic bryophytes.
\end{abstract}

Key words: diversity, epiphytes, indicator species, liverworts, mosses.

Guerra, G.,Arrocha, C., Rodríguez, G., Déleg, J., \& Benítez, Á. (2020). Briófitos en los troncos de árboles como indicadores de la alteración en bosques montanos de Panamá. Revista de Biología Tropical, 68(2), 492-502.

El Parque Nacional Volcán Barú se encuentra localizado mayormente en la ecorregión de los bosques montanos de Talamanca y en menor grado en la ecorregión de los bosques húmedos de Talamanca (Dinerstein et al., 1995). Los bosques montanos ocupan aproximadamente el $0.4 \%$ de la superficie de la Tierra, y soportan aproximadamente el 20 y $16 \%$ de la diversidad de plantas y vertebrados del planeta, respectivamente (Ray, Nair, Lawton, Welch, \& Pielke,
2006) y se consideran generalmente como puntos de alta biodiversidad (Hamilton, Juvik, \& Scatena, 1995; Doumenge, Gilmour, Pérez, \& Blochus, 1995). Estos sistemas, desempeñan importantes funciones hidrológicas, debido a que recogen humedad mediante la intercepción directa del agua líquida de las nubes orográficas, las nubes y la neblina arrastrada por el viento (Bruijnzeel \& Proctor, 1995; Cavelier, Solis, \& Jaramillo, 1996). También son de gran 
importancia ecológica como fuentes de agua y de otros servicios de los ecosistemas, para millones de personas que viven en los trópicos (Gradstein, Homeier, \& Gansert, 2008).

Los bosques lluviosos tropicales, especialmente los montanos, son ricos en briófitos epífitos (Frahm \& Gradstein, 1991; Frahm et al., 2003; Parolly \& Kürschner, 2004). Estos organismos evitan la erosión del suelo; fijan en sus estructuras enormes cantidades de $\mathrm{C} \mathrm{y}$ $\mathrm{N}$; ayudan a los bosques a mantener la humedad evitando así el estrés hídrico; y sirven de refugio, cobijo y alimentación a innumerables invertebrados y vertebrados (VanDerpoorten \& Goffinet, 2009). Además, acumulan materia orgánica para el establecimiento de plantas vasculares (Gradstein, Griffin, Morales, \& Nadkarni, 2001b).

Los briófitos son organismos poiquilohídricos, es decir, su humedad depende del medio ambiente (Salazar-Allen \& Chung, 2005) por ello se han utilizado como indicadores de alteraciones antrópicas en bosques montanos (Holz \& Gradstein, 2005; Nöske et al., 2008; Benítez, Prieto, \& Aragón, 2015). La alteración de los bosques produce cambios en las condiciones microclimáticas relacionados con la disponibilidad de humedad, luz y temperatura, dando como resultado que las epífitas de hábitats sombreados sean más vulnerables a dichos cambios, mientras que las briófitas de dosel son más tolerantes al estrés por desecación (Frahm et al., 2003). Adicionalmente, factores relacionados con los rasgos del hospedador (e.g. $\mathrm{DBH}$, textura de la corteza y $\mathrm{pH}$ ) son factores limitantes (Holz \& Gradstein, 2005; Benítez et al., 2015).

Los estudios relacionados con la ecología de briófitos de los bosques montanos tropicales de América Central y otras partes de la región son muy escasos (Wolf, 2005), y Panamá no es la excepción, donde se han realizado tres estudios utilizando briófitos como indicadores. Por ejemplo: el primero, enfocado a determinar cambios en la diversidad de briófitos a lo largo de un gradiente altitudinal (Gradstein \& Salazar-Allen, 1992); el segundo, relacionado con la riqueza y biomasa de epífitos vasculares y no vasculares en bosques montanos (Gómez, Rodríguez, Zotz, \& Bader, 2017); y el tercero, relacionado con cambios en la biomasa de briófitos y líquenes a lo largo de un gradiente en el Volcán Barú (Rodríguez-Quiel, MendietaLeiva, \& Bader, 2019). La mayor parte de estudios se han enfocado en epífitas vasculares como indicadoras de la alteración de los bosques en Panamá (Einzmann \& Zotz, 2016); por ende, este es el primer estudio en Panamá enfocado en evaluar el efecto de un gradiente de alteración sobre la riqueza y composición de briófitos epífitos en el Parque Nacional Volcán Barú, así como determinar especies indicadoras y que variables microclimáticas y de estructura del bosque influyen en la composición de estas comunidades.

\section{MATERIALES Y MÉTODOS}

Área de estudio: El estudio se llevó a cabo en la provincia de Chiriquí ubicada al occidente de la República de Panamá, específicamente en el Parque Nacional Volcán Barú, que es un bosque perennifolio ombrófilo tropical latifoliado (BPOTL) altimontano (Autoridad Nacional del Ambiente, 2010). La precipitación media anual varía entre 3700 y $2100 \mathrm{~mm}$ y una temperatura promedio anual de $12.5^{\circ} \mathrm{C}$ (ANAM, 2010). Este bosque es el tipo de vegetación más extensa en el PNVB y se ubica entre los 1800 a $2300 \mathrm{~m}$. Se caracteriza por presentar un dosel alto, con 35-45 m de altura y árboles emergentes que llegan a los $50 \mathrm{~m}$. Generalmente, el sotobosque está compuesto de grupos de arbustos y pocas hierbas bajas. Es muy común la presencia de helechos arborescentes, palmas y carricillos (Chusquea sp.); en áreas cercanas a los claros los arbustos están cubiertos con abundantes trepadoras.

El sitio específico del estudio es por la ladera occidental, en Paso Ancho, se evaluaron dos áreas boscosas con distinto grado de intervención, la primera ubicada a $08^{\circ} 48^{\prime} 55.13^{\prime \prime}$ $\mathrm{N}$ - 82³4'33.50" W a una elevación de $2047 \mathrm{~m}$. conocida como el bosque de las Brujas y la segunda área ubicada a $08^{\circ} 48^{\prime} 51.28^{\prime}$ ' 


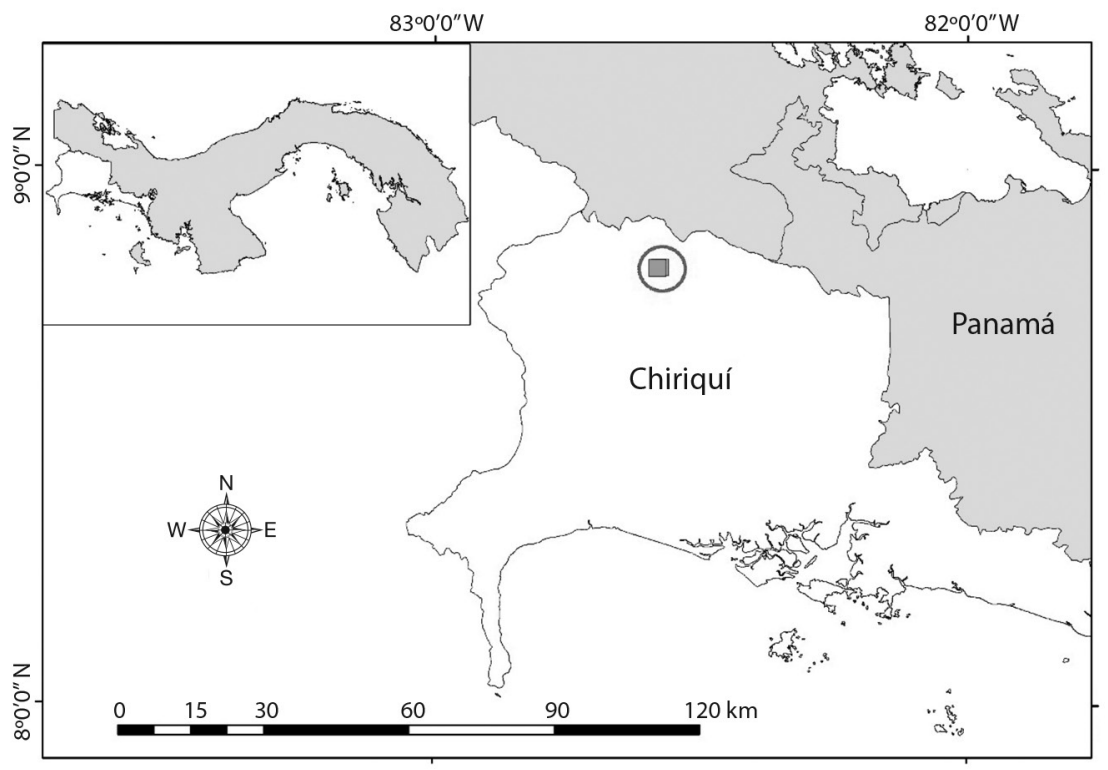

Fig. 1. Área de estudio en los bosques montanos del Volcán Barú, Panamá. Fig. 1. Study area on the montane forest of Baru Volcano, Panama.

$\mathrm{N}-82^{\circ} 34^{\prime} 50.68$ " W a una elevación de $1900 \mathrm{~m}$ llamada zona de pastizal (Fig. 1).

El bosque no alterado (Bosque de las Brujas), a menudo se cubre de niebla, la vegetación es densa y alta, suelo cubierto de una capa densa de hojarasca, gran cobertura de epífitas vasculares. La entrada de luz promedio es de $39.04 \%$, temperatura promedio de $17.57{ }^{\circ} \mathrm{C} \mathrm{y}$ humedad relativa promedio de $71.50 \%$.

El bosque alterado (Zona de pastizal), con alto grado de intervención, vegetación dominada por pastizales, predominan las plantas de la familia Poaceae, pinos y ericáceas de baja estatura con árboles aislados. La entrada de luz promedio es de $46.89 \%$, temperatura promedio de $18.67{ }^{\circ} \mathrm{C}$ y humedad relativa promedio de $69.92 \%$.

Diseño y recolección de datos: En cada bosque se delimitaron diez parcelas de $5 \times 5 \mathrm{~m}$, donde se seleccionaron dos árboles que tuviesen $>10 \mathrm{~cm}$ DAP (DAP a una altura de $1.3 \mathrm{~m}$ ). En cada árbol se registró la frecuencia y cobertura de briófitos en un cuadrante de $20 \times 30 \mathrm{~cm}$, a dos alturas (base y altura de pecho) y en dos orientaciones. El estudio se llevó a cabo de agosto 2016 a marzo 2017.

Para registrar temperatura y humedad se colocó un Dataloggers Easy Log EL-USB2-LCD en cada parcela. Para medir la incidencia de luz por parcela se utilizó un densiómetro esférico convexo (modelo C No. 43887), a 1 $\mathrm{m}$ de distancia desde el tronco del árbol seleccionado, en dirección norte, sur, este y oeste. Posteriormente se contó la cantidad de puntos con entrada de luz, que se multiplicó por 1.04 para obtener el porcentaje de la superficie no ocupada por el dosel (Lemmon, 1957; Mandl, Kessler, \& Gradstein, 2009).

Para las mediciones de $\mathrm{pH}$ de cada árbol, se pesaron en una balanza digital dos gr de corteza previamente secada, luego se añadieron $40 \mathrm{ml}$ de agua destilada, se agitó suavemente con un policial y se dejó humedecer completamente durante una hora con agitación ocasional para asegurarse de que la muestra estuviera bien mezclada. Mientras las muestras se empapaban se calibró el medidor de $\mathrm{pH}$ frente a los tampones de $\mathrm{pH} 7, \mathrm{pH} 4$ y $\mathrm{pH}$ 14. Después de transcurrida 1 hora se dio una agitación suave final con el policial y se insertó la sonda de 
electrodo de $\mathrm{pH}$ enjuagada con agua destilada, se revolvió suavemente con la sonda y se dejó estabilizar durante 2 minutos antes de registrar la lectura. Se repitió el procedimiento con cada muestra y se enjuagó la sonda del electrodo con agua destilada entre cada lectura (Soto-Medina, Lücking, \& Bolaños-Rojas, 2012).

Para la identificación de las muestras se utilizó un microscopio Zeiss PRIMO STAR y un estéreo microscopio Zeiss Stemi DV4 y se utilizaron diversas claves taxonómicas (Gradstein, 1989, 2016; Allen, 1994, 2002, 2010, 2018; Gradstein, Churchill, \& Salazar-Allen, 2001a; Costa, 2008; Gradstein \& Ilkiu-Borges, 2009). Las muestras identificadas fueron depositadas en el Herbario de la Universidad Autónoma de Chiriquí (UCH) y se colectaron bajo permiso de colecta $\mathrm{N}^{\circ}$. SE/P-4-17, emitido por el Ministerio de Ambiente de Panamá.

Análisis de datos: Se estimó la riqueza de especies por tipo de bosque con una curva de rarefacción basado en muestras y el estimador no paramétrico Chao 2. Adicionalmente, se evaluó la diversidad con los índices de Simpson y Shannon Weaver. Para analizar los efectos de variables ambientales como luz, temperatura, humedad, DBH y $\mathrm{pH}$ sobre la riqueza de briófitos epífitos se realizó un Modelo Lineal Generalizado (GLM) con una distribución de errores "Poisson" y función de vínculo logarítmica (Zuur, Ieno, \& Smith, 2007).

Se realizó un análisis de escalamiento multidimensional no métrico (NMDS) para tratar de representar en un espacio geométrico de pocas dimensiones las proximidades existentes entre la composición de especies de briófitos en los diferentes tipos de bosques, para lo cual se utilizó la distancia Bray-Curtis y 999 permutaciones de Monte Carlo. Finalmente, se realizó una correlación entre los dos ejes ajustados y las variables ambientales (luz, temperatura, humedad, DAP y pH), con la función "envfit". Para evaluar la similitud de especies entre los dos tipos de bosques se realizó un análisis multivariado basado en permutaciones por pares (Anderson, Gorley, \& Clarke, 2008). Adicionalmente, aplicamos un análisis de especies indicadoras (ISA) para determinar que especies son las mejores indicadoras en cada uno de los tipos de bosques (Dufrêne \& Legendre, 1997). El valor de indicación varía de 0 (cuando una especie estuvo ausente en un tipo de bosque) a 100 (cuando la especie ocurrió en todos los árboles de un tipo de bosque y estuvo ausente en el otro tipo de bosque). Todos los análisis se

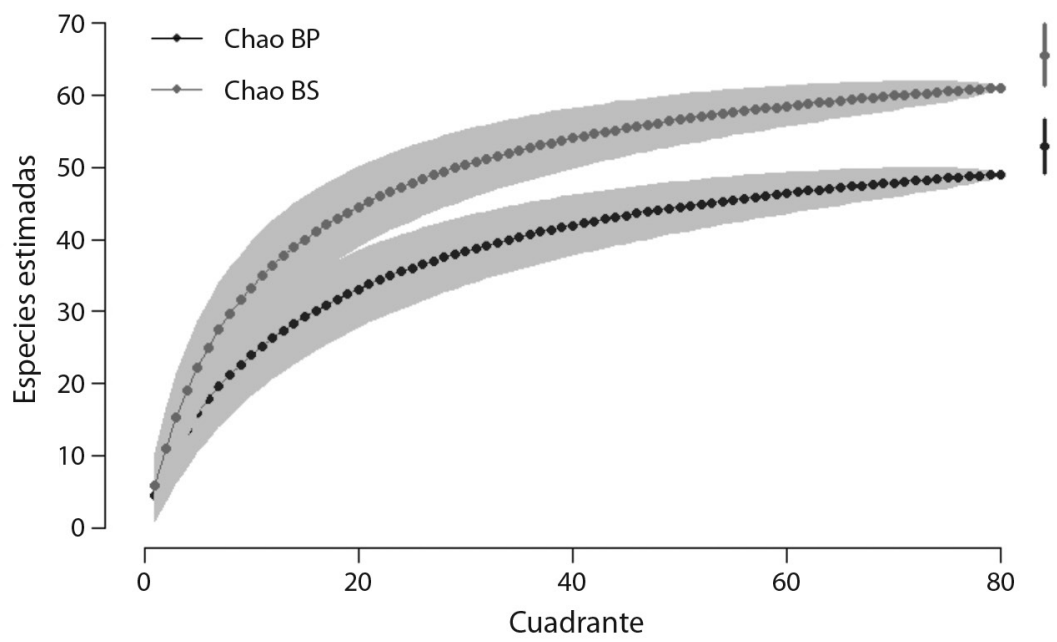

Fig. 2. Curva de rarefacción de especies con el $95 \%$ de intervalo de confianza y estimador Chao 2 (puntos a la derecha de la figura) de los dos bosques estudiados.

Fig. 2. Rarefaction curves with $95 \%$ confidence intervals and Chao 2 estimator (points in the right of figure) of two study forests. 
TABLA 1

Resultados del Modelo Lineal Generalizado entre la riqueza de briófitos y las variables ambientales

TABLE 1

Results of the Generalized Linear Model showing the effects of environmental factors on the epiphytic bryophytes richness

\begin{tabular}{|c|c|c|c|c|}
\hline Variables & Estimador & SE & $\mathrm{Z}$ & $\mathrm{P}$ \\
\hline $\mathrm{DBH}$ & -0.0029 & 0.0031 & -0.9410 & 0.3469 \\
\hline Luz & -0.0004 & 0.0054 & -0.0680 & 0.9458 \\
\hline Temperatura & -0.2349 & 0.1559 & -1.5060 & 0.1320 \\
\hline Humedad & 0.0080 & 0.0262 & 0.3040 & 0.7614 \\
\hline $\mathrm{pH}$ & -0.0043 & 0.0505 & -0.0850 & 0.9324 \\
\hline Bosque alterado (BS) & 0.5438 & 0.1546 & 3.5170 & 0.0004 \\
\hline Bosque no alterado (BP) & 5.3026 & 4.2101 & 1.2590 & 0.2079 \\
\hline
\end{tabular}

realizaron con el programa $\mathrm{R}$ y el paquete estadístico "vegan" (Oksanen et al., 2019).

\section{RESULTADOS}

Diversidad alfa: Se registraron un total de 86 especies (49 hepáticas y 37 musgos) en 160 cuadrantes. La curva de rarefacción de especies y el estimador de riqueza Chao 2 (Fig. 2) señalaron un mayor número de especies estimadas para los bosques alterados (BS) en comparación con los bosques no alterados (BP). La riqueza de especies y los índices de diversidad (Shannon-Weaver y Simpson) presentaron una tendencia mayor para el bosque alterado en comparación con el bosque no alterado. Así, para los bosques no alterados el rango mínimo y máximo fueron de dos y 9 especies, respectivamente. Mientras que para los bosques alterados el valor mínimo y máximo fue de dos y 13 especies respectivamente. En cuanto a los índices de diversidad, el valor medio del índice de Shannon-Weaver fue de 1.40 y 1.70 para el bosque no alterado y bosque alterado, respectivamente. Similar tendencia señalo el índice de Simpson con valores similares para los dos tipos de bosques ( 0.50 para bosques no alteados y 0.58 para los bosques alterados).

El GLM señaló que la riqueza de especies estuvo influenciada por el tipo de bosque, donde el bosque alterado indicó un efecto positivo, las demás variables microclimáticas y rasgos del hospedador no fueron influyentes (Tabla 1).

Diversidad beta y especies indicadoras: El análisis NMDS señaló un claro ordenamiento entre las comunidades de briófitos relacionadas con la alteración de los bosques. Las comunidades de bosques primarios estuvieron limitadas por una mayor humedad, mientras que en los bosques alterados por la luz y la temperatura fueron factores limitantes (Fig. 3, Tabla 2).

El tipo de bosque explica la mayor variabilidad en la composición de las comunidades de briófitos epífitos con el $47 \%$, seguido de temperatura con el $44 \%$, luz con el $25 \%$, $\mathrm{pH}$ con el $20 \%$ y humedad con el $14 \%$. Así mismo, el PERMANOVA por pares reveló diferencias significativas $(p=0.001)$ en la composición de briófitos epífitos por cobertura entre los dos tipos de bosques con un grado de disimilitud del 97.87. Se reportaron 12 especies indicadoras para los bosques no alterados, donde Meteoridium remotifolium, Plagiochila simplex y Plagiochila diversifolia presentaron los valores más altos de indicación. Mientras que para los bosques alterados las 24 especies pertenecieron a la familia Lejeuneaceae y Frullaniaceae (Apéndice 1). 
TABLA 2

Coeficientes de correlación de los factores ambientales ajustados a los dos primeros ejes de la ordenación NMDS

TABLE 2

Squared correlation coefficients $\left(\mathrm{r}^{2}\right)$ fitted on the first two axes of the NMDS ordination for environmental factors

\begin{tabular}{|c|c|c|c|c|}
\hline Factores & NMDS1 & NMDS2 & $r^{2}$ & $P$ \\
\hline Tipo de bosque & & & 0.477 & $<0.001$ \\
\hline Bosque no alterado & -0.466 & 0.013 & & \\
\hline Bosque alterado & 0.472 & -0.012 & & \\
\hline DBH & 0.601 & -0.799 & 0.094 & $<0.001$ \\
\hline Temperatura & 0.999 & 0.046 & 0.449 & $<0.001$ \\
\hline Humedad & -0.978 & 0.208 & 0.142 & $<0.001$ \\
\hline Luz & 0.808 & -0.589 & 0.252 & $<0.001$ \\
\hline $\mathrm{pH}$ & 0.097 & 0.995 & 0.201 & $<0.001$ \\
\hline
\end{tabular}

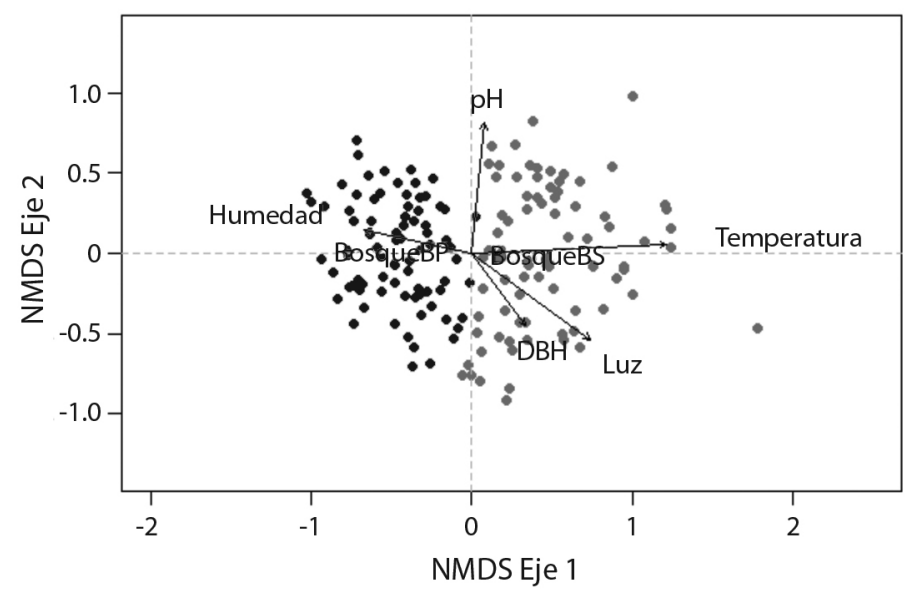

Fig. 3. Escalamiento Multidimensional No Métrico (NMDS) de la composición de especies y las variables ambientales en los dos tipos de bosques montanos estudiados.

Fig. 3. Non-metric multidimensional scaling analysis of species composition and environmental variables in the two studied montane forests. Undisturbed forest (green colour) and disturbed forest (blue color).

\section{DISCUSIÓN}

Los resultados indicaron que los bosques montanos del volcán Barú son muy diversos, y mostraron cambios en la riqueza y composición de las comunidades de briófitos epífitos, debido a la alteración de los bosques que implica cambios en el microclima (humedad, luz y temperatura). Además, rasgos del hospedador como el diámetro de los árboles y el pH condicionaron las comunidades. Las 86 especies de briófitos reportados en el sotobosque fue alta al comparar con el estudio realizado en bosques montanos tropicales de Ecuador por Benítez et al. (2015) donde reportan un total de 67 especies y el de Gradstein \& Culmsee (2010) con 58 especies en bosques montanos de Indonesia, así podemos señalar que bosques montanos del volcán Barú mantienen una gran diversidad de briófitos en el sotobosque.

De manera general se ha documentado una disminución en la riqueza de briófitos a medida que se encuentra más perturbado el bosque, porque se ve afectada la humedad, la temperatura y las condiciones de luz (Wolf, 2005; Gradstein et al., 2008; Gradstein \& Sporn, 2010; 
Nöske et al., 2008; Benítez et al., 2015). Sin embargo, en este estudio se registró una mayor riqueza de especies en bosques alterados, debido a que ocurre un reemplazo de las especies sensibles por aquellas especies que están adaptadas a condiciones climáticas de mayor luz y temperatura (epífitos de sol). En concordancia con nuestros resultados aquí obtenidos Holz \& Gradstein (2005), encontraron que la riqueza de especies en bosque no alterados (primarios) y secundarios (alterados) es prácticamente la misma, o incluso ligeramente mayor en los bosques secundarios.

Las comunidades se ordenaron en función del gradiente de alteración relacionado con cambios en el microclima y rasgos del hospedador. Bajo esta premisa se ha documentado que el establecimiento y la formación de comunidades de briófitos están condicionados a gradientes microclimáticos de humedad y luz (Frahm et al., 2003; Holz \& Gradstein, 2005; Sporn, Bos, Hoffstätter-Müncheberg, Kessler, \& Gradstein, 2009; Nöske et al., 2008; Benítez et al., 2015). En los bosques alterados se evidenció una dominancia de epífitas de sol, debido a que están adaptadas a condiciones de mayor luz y temperatura. Por ejemplo, las hepáticas de la familia Frullaniaceae (Frullania apiculata, F. arecae, F. ericoides y Lejeuneaceae (Acanthocoleus aberrans, Brachiolejeunea laxifolia y Cheilolejeunea holostipa), así como los musgos Cryphaea patens y Cyrtohypnum sharpii fueron dominantes y presentaron los valores más altos de indicación. De manera similar varios estudios han documentados que este grupo de especies están mejor adaptadas a condiciones de mayor entrada de luz y menor humedad (Holz \& Gradstein, 2005; Nöske et al., 2008; Gradstein et al., 2001a; Benítez et al., 2015). En cambio, en el bosque no alterado los epífitos de sombra de la familia Plagiochilaceae (Plagiochila diversifolia, Plagiochila laetevirens y Plagiochila simplex) y los musgos Meteoridium remotifolium, Platygyriella densa y Porotrichodendron lindigii adaptadas a condiciones de mayor humedad y menor disponibilidad de luz fueron dominantes y presentaron valores altos de indicación en el sotobosque de los bosques no alterados (Acebey, Gradstein, \& Krömer, 2003; Gradstein et al., 2008, Benítez et al., 2015).

Los rasgos del hospedador relacionados con el diámetro de los árboles y el $\mathrm{pH}$ influyeron directamente en la composición de las comunidades. En este contexto varios estudios han documentado que el $\mathrm{pH}$ es un factor determinante en las comunidades de briófitos epífitos. Frahm et al. (2003) y Pereira, Müller, \& Moya (2014) demostraron que los briófitos tienen preferencia por el $\mathrm{pH}$ cercano a la neutralidad, como en nuestro caso el promedio del $\mathrm{pH}$ estuvo entre los valores de 5 a 7 . Por otra parte, el diámetro de los árboles fue un factor limitante en las comunidades de briófitos epífitos, que según otros estudios está relacionado con una mayor superficie para los procesos de colonización de las especies (Gradstein \& Culmsee, 2010; Benítez et al., 2015).

Se concluye que la riqueza de especies de briófitos es alta en los bosques montanos del volcán Barú en Panamá. Así mismo, las comunidades de briófitos fueron indicadores efectivos de la alteración de los bosques montanos relacionados con cambios en la humedad, luz, temperatura y rasgos del hospedador. Las comunidades de epífitos de sombra fueron afectadas directamente por cambios en el microclima de los bosques alterados, por ello la conservación de estos bosques no alterados garantizará la conservación de estas especies con altos requerimientos de humedad.

\section{AGRADECIMIENTOS}

A la Secretaría Nacional de Ciencia y Tecnología de Panamá (SENACYT) por el financiamiento parcial de este trabajo a través del proyecto APY-GC-2017B-034 y a la Vicerrectoría de Investigación y Posgrado de la Universidad Autónoma de Chiriquí que auspició parcialmente el trabajo de campo por medio del programa de subsidio a las actividades de investigación de la UNACHI. 


\section{RESUMEN}

Introducción: Los bosques montanos tropicales se caracterizan por una alta riqueza y endemismo de briófitos. Sin embargo, la diversidad es altamente sensible a cambios microclimáticos relacionados con alteraciones antrópicas. Objetivo: En este estudio, se evaluó la riqueza y composición de briófitos epífitos en los troncos de árboles de bosques montanos alterados y bosques no alterados ubicados en la ladera occidental del Parque Nacional Volcán Barú. Métodos: Los bosques no alterados mostraron alta humedad, mientras que los bosques alterados mostraron altos niveles de luz y temperatura. En cada árbol evaluamos la presencia y cobertura de briófitos epífitos en 160 cuadrantes de $20 \times 30 \mathrm{~cm}$. Resultados: Se registraron un total de 86 especies (49 hepáticas y 37 musgos). La riqueza fue positivamente influenciada por la alteración antrópica, donde los bosques alterados presentaron un mayor número de epífitos de sol. La composición de las comunidades difiere entre los dos tipos de bosques, donde las epífitas de sombra fueron restringidas a bosques no alterados, mientras que las epífitas de sol a zonas alteradas. Conclusiones: Las comunidades de briófitos fueron indicadores efectivos de la alteración de los bosques montanos del Parque Nacional Volcán Barú. Por lo tanto, la conservación de los bosques no alterados garantizará la conservación de una rica y diversa comunidad de briófitos epífitos.

Palabras clave: diversidad, epífitos, especies indicadoras, hepáticas, musgos.

\section{REFERENCIAS}

Acebey, A., Gradstein, S.R., \& Krömer, T. (2003). Species richness and habitat diversification of bryophytes in submontane rain forest and fallows of Bolivia. Journal of Tropical Ecology, 19, 9-18.

Anderson, M.J., Gorley, R.N., \& Clarke, K.R. (2008). PERMANOVA+ for PRIMER: Guide to Software and Statistical Methods. Plymouth, UK: PRIMER-E.

Allen, B.H. (1994). Moss Flora of Central America. Part 1. Sphagnaceae-Calymperaceae. Missouri: Missouri Botanical Garden Press.

Allen, B.H. (2002). Moss Flora of Central America. Part 2. Encalyptaceae-Orthotrichaceae. Missouri: Missouri Botanical Garden Press.

Allen, B.H. (2010). Moss Flora of Central America. Part 3. Anomodontaceae-Symphyodontaceae. Missouri: Missouri Botanical Garden Press.

Allen, B.H. (2018). Moss flora of Central America. Part 4. Fabroniaceae-Polytrichaceae. Missouri: Missouri Botanical Garden Press.
ANAM (Autoridad Nacional del Ambiente) (2010). Atlas Ambiental de la República de Panamá. Primera versión. Panamá.

Benítez, Á., Prieto, M., \& Aragón, G. (2015). Large trees and dense canopies: key factors for maintaining high epiphytic diversity on trunk bases (bryophytes and lichens) in tropical montane forests. Forestry, 88, 521-527.

Bruijnzeel, L., \& Proctor, J. (1995). Hydrology and biogeochemistry of tropical montane cloud forest: What do we really know? En L. Hamilton, J. Juvik, \& F. Scatena (Eds.). Tropical Montane Cloud Forests (pp. 38-78). New York: Springer.

Cavelier, J., Solis, D., \& Jaramillo, M. (1996). Fog interception in montane forest across the Central cordillera of Panama. Journal of Tropical Ecology, 12, 357-369.

Costa, D. (2008). Metzgeriaceae (Hepaticae). Flora Neotropica Monograph 102. New York, EE.UU.: New York Botanical Press.

Dinerstein, E., Olson, D., Graham, D., Webster, A., Primm, S., Bookbinder, M., \& Ledec, G. (1995). Una evaluación del estado de conservación de las ecorregiones terrestres de América Latina y el Caribe. Washington, D.C., EE.UU.: Banco Mundial.

Doumenge, C., Gilmour, D., Pérez, M.R., \& Blochus, J. (1995). Tropical montane cloud forests: Conservation status and management issues. En L. Hamilton, J. Juvik, \& F. Scatena (Eds.), Tropical Montane Cloud Forests (pp. 24-37). New York: Springer.

Dufrêne, M., \& Legendre, P. (1997). Species assemblages and indicator species: the need for a flexible asymmetrical approach. Ecological Monographs, 67, 345-366.

Frahm, J., \& Gradstein, R. (1991). An altitudinal zonation of tropical rain forests using bryophytes. Journal of Biogeography, 18, 669-678.

Frahm, J., O’Shea, B., Pócs, T., Koponen, T., Piippo, S., Enroth, J., Rao, P., \& Fang, Y.M. (2003). Manual of tropical bryology. Tropical Bryology, 23, 1-196.

Gómez, D.C., Rodríguez Quiel, C., Zotz, G., \& Bader, M.Y. (2017). Species richness and biomass of epiphytic vegetation in a tropical montane forest in western Panama. Tropical Conservation Science, 10, 1-17.

Gradstein, S.R. (1989). A key to the Hepaticae and Anthocerotae of Puerto Rico and the Virgin Islands. The Bryologist, 92, 329-348.

Gradstein, S.R., \& Culmsee, H. (2010). Bryophyte diversity on tree trunks in montane forests of Central Sulawesi, Indonesia. Tropical Bryology, 31, 95-105. 
Gradstein, S.R., \& Salazar-Allen, N. (1992). Bryophyte diversity along an altitudinal gradient in Darien National Park, Panama. Tropical Bryology, 5, 61-71.

Gradstein, S.R., Churchill, S., \& Salazar-Allen, N. (2001a). Guide to the bryophytes of Tropical America. Memoirs of the New York Botanical Garden. New York, EE.UU.: New York Botanical Garden Press.

Gradstein, S.R., Griffin, D., Morales, M., \& Nadkarni, N. (2001b). Diversity and habitat differentiation of mosses and liverworts in the cloud forest of Monteverde, Costa Rica. Caldasia, 23, 203-212.

Gradstein, S.R., Homeier, J., \& Gansert, D. (2008). The tropical mountain forest: Patterns and Processes in a biodiversity hotspot. Göttingen, Alemania: Universitätsverlag Göttingen.

Gradstein, S.R., \& Ilkiu-Borges, A.L. (2009). Guide to the plants of Central French Guiana. Part IV. Liverworts and Hornworts. Memoirs of the New York Botanical Garden. New York, EE.UU.: New York Botanical Garden Press.

Gradstein, S.R., \& Sporn, S.G. (2010). Diversity of epiphytic bryophytes along land use gradients in the Tropics. Nova Hedwigia Beiheft, 138, 309-321.

Gradstein, S.R. (2016). The genus Plagiochila (Marchantiophyta) in Colombia. Revista de la Academia Colombiana de Ciencias Exactas, Físicas y Naturales, 40, 104-136.

Einzmann, H.J.R., \& Zotz, G. (2016). How diverse are epiphyte assemblages in plantations and secondary forests in tropical lowlands? Tropical Conservation Science, 9(2), 629-647.

Hamilton, L., Juvik, J., \& Scatena, F. (1995). The Puerto Rico Tropical Cloud Forest Symposium: Introduction and Workshop Synthesis. En L. Hamilton, J. Juvik, \& F. Scatena (Eds.), Tropical Montane Cloud Forests (pp. 1-18). New York: Springer.

Holz, I., \& Gradstein, R. (2005). Cryptogamic epiphytes in primary and recovering upper montane oak forests of Costa Rica - species richness, community composition and ecology. Plant Ecology, 178, 89-109.

Lemmon, P. (1957). A new instrument for measuring forest overstory density. Journal of Forestry, 55, 667-668.

Mandl, N.A., Kessler, M., \& Gradstein, S.R. (2009). Effects of environmental heterogeneity on species diversity and composition of terrestrial bryophyte assemblages in tropical montane forests of southern Ecuador. Plant Ecology \& Diversity, 2(3), 313-321.

Nöske, N., Hilt, N., Welner, F., Brehm, G., Fiedler, K. Sipman, H., \& Gradstein, S.R. (2008). Disturbance effects on diversity of epiphytes and moths in a montane forest in Ecuador. Basic and Applied Ecology, 9, 4-12.
Oksanen, J., Blanchet, F.G., Kindt, R., Legendre, P., Minchin, P.R., O’Hara, R.B., Simpson, G.L., Solymos, P., Stevens, M.H.H., \& Wagner, H. (2019). Vegan: Community Ecology Package ( R Package version 2.5-6). Recuperado de https://cran.r-project.org/web/ packages/vegan/vegan.pdf

Parolly, G., \& Kürschner, H. (2004). Ecosociological studies in Ecuadorian bryophyte communities. II. Syntaxonomy of the submontane and montane epiphytic vegetation of S Ecuador. Nova Hedwigia, 79, 377-424.

Pereira, I., Müller, F., \& Moya, M. (2014). Influence of Nothofagus $\mathrm{pH}$ on lichen and bryophytes richness, Central Chile. Guyana Botánica, 71(1), 120-130.

Ray, D., Nair, U., Lawton, R., Welch, R., \& Pielke, R. (2006). Impact of land use on Costa Rican tropical montane cloud forests: Sensitivity of orographic cloud formation to deforestation in the plains. Journal of Geophysical Research, 111, 1-16.

Rodríguez-Quiel, E.E., Medieta-Leiva, G., \& Bader, M.Y. (2019). Elevational patterns of bryophyte and lichen biomass differ among substrates in the tropical montane forest of Baru Volcano, Panama. Journal of Bryology, 41(2), 95-106.

Salazar-Allen, N., \& Chung, C. (2005). Briófitas (antocerotes, hepáticas y musgos) de la Región de Bahía Honda (Veraguas, Panamá). En S. Castroviejo \& A. Ibáñez (Eds.), Estudios sobre la Biodiversidad de da Región de Bahía Honda (Veraguas, Panamá) (pp. 95-176). Madrid: Consejo Superior de Investigaciones Científicas.

Soto-Medina, E., Lücking, R., \& Bolaños-Rojas, A. (2012). Especificidad de forófito y preferencias microambientales de los líquenes cortícolas en cinco forófitos del bosque premontano de finca Zíngara, Cali, Colombia. Revista de Biología Tropical, 60, 843-856.

Sporn, S., Bos, M., Hoffstätter-Müncheberg, M., Kessler, M., \& Gradstein, S.R. (2009). Microclimate determines community composition but not richness of epiphytic understory bryophytes of rainforest and cacao agroforests in Indonesia. Functional Plant Biology, 36, 171-179.

VanDerpoorten, A., \& Goffinet, B. (2009). Introduction to bryophytes. Cambridge, Reino Unido: Cambridge University Press.

Wolf, J.H.D. (2005). The response of epiphytes to anthropogenic disturbance of pine-oak forests in the highlands of Chiapas, Mexico. Forest Ecology and Management, 212, 376-396.

Zuur, A.F., Ieno, E.N., \& Smith, G.M. (2007). Analysing ecological data. New York EE.UU.: Springer. 


\section{APÉNDICE 1}

Especies de briófitos que aparecen en los dos tipos de bosques y especies indicadoras para cada tipo de bosque

\section{APPENDIX 1}

Bryophyte species and Indicator species with an indicator value for each forest type

\begin{tabular}{|c|c|c|c|c|c|}
\hline Especies & $\begin{array}{c}\text { Bosque no } \\
\text { alterado (BP) }\end{array}$ & $\begin{array}{c}\text { Bosque } \\
\text { Alterado (BS) }\end{array}$ & $\begin{array}{c}\text { Tipo } \\
\text { de bosque }\end{array}$ & $\begin{array}{c}\text { Valor de } \\
\text { indicación (\%) }\end{array}$ & P-valor \\
\hline \multicolumn{6}{|l|}{ Musgos } \\
\hline Actinodontium sprucei (Mitt.) A. Jaeger & 0 & 1 & BS & 8.80 & 0.011 \\
\hline Aerolindigia capillacea (Hornsch.) M. Menzel & 1 & 0 & $\mathrm{BP}$ & 1.20 & 1.000 \\
\hline Brachymenium systylium (Müll. Hal.) A. Jaeger & 0 & 1 & BS & 8.80 & 0.013 \\
\hline Brachythecium ruderale (Brid.) W.R. Buck & 0 & 1 & BS & 6.20 & 0.065 \\
\hline Chryso-hypnum diminutivum (Hampe) W.R. Buck & 1 & 1 & $\mathrm{BP}$ & 9.30 & 0.832 \\
\hline $\begin{array}{l}\text { Cyrto-hypnum sharpii (H.A. Crum) W.R. Buck \& } \\
\text { H.A. Crum }\end{array}$ & 0 & 1 & $\mathrm{BS}$ & 28.70 & 0.000 \\
\hline Cryphaea patens Hornsch ex Müll. Hal. & 0 & 1 & BS & 20.00 & 0.000 \\
\hline Cryphaeasp. & 0 & 1 & BS & 5.00 & 0.119 \\
\hline Daltonia trachyodonta Mitt. & 0 & 1 & BS & 3.80 & 0.247 \\
\hline Encalypta flowersiana Horton & 0 & 1 & BS & 2.50 & 0.494 \\
\hline Entodon hampeanus Müll. Hal. & 0 & 1 & BS & 21.20 & 0.000 \\
\hline Fissidens crispus Mont. & 0 & 1 & BS & 1.20 & 1.000 \\
\hline Groutiella chimborazensis (Spruce ex Mitt.) Florsch. & 1 & 1 & BS & 12.90 & 0.008 \\
\hline Leptodontium excelsum (Süll) Britt. & 1 & 1 & $\mathrm{BS}$ & 17.40 & 0.000 \\
\hline Leptodontium ulocalyx (Müll. Hal.) Mitt. & 0 & 1 & BS & 5.00 & 0.118 \\
\hline Leucodon curvirostris Hampe & 1 & 0 & $\mathrm{BP}$ & 3.80 & 0.241 \\
\hline Meteoridium remotifolium (Müll. Hal.) Manuel & 1 & 0 & BP & 82.50 & 0.000 \\
\hline Meteorium deppei (Hornsch. ex Müll. Hal.) Mitt. & 1 & 1 & BS & 2.00 & 0.994 \\
\hline Meteorium teres Mitt. & 1 & 0 & $\mathrm{BP}$ & 2.50 & 0.504 \\
\hline Neckera ehrenbergii Müll. Hal. & 1 & 1 & $\mathrm{BP}$ & 15.00 & 0.033 \\
\hline Orthostichella rigida (Müll. Hal.) B.H. Allen \& Magill & 1 & 1 & BS & 2.80 & 0.999 \\
\hline $\begin{array}{l}\text { Orthostichella versicolor (Müll. Hal.) B.H. Allen \& } \\
\text { W.R. Buck }\end{array}$ & 1 & 1 & $\mathrm{BS}$ & 25.10 & 0.075 \\
\hline Platygyriella densa (Hook.) W.R. Buck & 1 & 1 & BS & 21.40 & 0.000 \\
\hline Porotrichodendron lindigii (Hampe) W.R. Buck & 1 & 0 & BP & 7.50 & 0.027 \\
\hline Prionodon densus (Sw. ex Hedw.) Müll. Hal. & 1 & 0 & BP & 1.20 & 1.000 \\
\hline Racopilum tomentosum (Hedw.) Brid. & 1 & 1 & $\mathrm{BS}$ & 2.80 & 0.628 \\
\hline Rhynchostegium scariosum (Taylor) A. Jaeger & 0 & 1 & BS & 11.20 & 0.005 \\
\hline Sematophyllum adnatum (Michx.) E. Britton & 0 & 1 & BS & 7.50 & 0.029 \\
\hline $\begin{array}{l}\text { Sematophyllum swartzii (Schwägr.) W.H. Welch \& } \\
\text { H.A. Crum }\end{array}$ & 0 & 1 & $\mathrm{BS}$ & 2.50 & 0.493 \\
\hline Sematophyllum virgatum B.H. Allen & 0 & 1 & BS & 1.20 & 1.000 \\
\hline Squamidium livens (Schwägr.) Broth. & 0 & 1 & BS & 5.00 & 0.120 \\
\hline Streptopogon calymperes Müll. Hal. & 0 & 1 & BS & 6.20 & 0.055 \\
\hline Taxiphyllum scalpellifolium (Müll. Hal.) Broth. & 1 & 0 & BP & 7.50 & 0.027 \\
\hline Thuidium delicatulum (Hedw.) Schimp. & 1 & 0 & $\mathrm{BP}$ & 16.20 & 0.000 \\
\hline Toloxis imponderosa (Taylor) W.R. Buck & 1 & 0 & $\mathrm{BP}$ & 6.20 & 0.055 \\
\hline Trichostomum tenuirostre (Hook. \& Taylor) Lindb. & 1 & 0 & BP & 3.80 & 0.252 \\
\hline Zygodon viridissimus (Dicks.) Brid. & 1 & 1 & $\mathrm{BP}$ & 6.90 & 1.000 \\
\hline \multicolumn{6}{|l|}{ Hepáticas } \\
\hline Acanthocoleus aberrans (Lindenb. \& Gottsche) Kruijt. & 1 & 1 & BS & 44.90 & 0.000 \\
\hline $\begin{array}{l}\text { Anoplolejeunea conferta C.F.W. Meissn. ex Spreng.) } \\
\text { A. Evans }\end{array}$ & 0 & 1 & $\mathrm{BS}$ & 5.00 & 0.121 \\
\hline Aphanolejeunea sp. & 1 & 0 & $\mathrm{BP}$ & 1.20 & 1.000 \\
\hline
\end{tabular}




\begin{tabular}{|c|c|c|c|c|c|}
\hline Especies & $\begin{array}{c}\text { Bosque no } \\
\text { alterado (BP) }\end{array}$ & $\begin{array}{c}\text { Bosque } \\
\text { Alterado (BS) }\end{array}$ & $\begin{array}{l}\text { Tipo de } \\
\text { bosque }\end{array}$ & $\begin{array}{c}\text { Valor de } \\
\text { indicación (\%) }\end{array}$ & P-valor \\
\hline Archilejeunea parviflora (Nees) Steph. & 1 & 0 & BP & 1.20 & 1.000 \\
\hline Archilejeuneasp & 1 & 0 & $\mathrm{BP}$ & 2.50 & 0.503 \\
\hline Brachiolejeunea laxifolia (Taylor) Schiffn. & 0 & 1 & BS & 18.70 & 0.000 \\
\hline Brachiolejeunea sp. & 0 & 1 & BS & 3.80 & 0.239 \\
\hline Cheilolejeunea filiformis (Sw.) W.Ye et al. & 0 & 1 & BS & 1.20 & 1.000 \\
\hline Cheilolejeunea holostipa (Spruce) Grolle \& R. L. Zhu & 1 & 1 & BS & 20.20 & 0.045 \\
\hline Cheilolejeunea sp. & 0 & 1 & BS & 3.80 & 0.247 \\
\hline Cheilolejeunea sp 1 & 0 & 1 & BS & 10.00 & 0.007 \\
\hline Dicranolejeunea axilaris (Nees \& Mont.) & 0 & 1 & BS & 2.50 & 0.500 \\
\hline Frullania apiculata (Reinw., Blume \& Nees) Nees & 1 & 1 & BS & 17.60 & 0.004 \\
\hline Frullania arecae (Spreng.) Gottsche & 0 & 1 & BS & 13.70 & 0.001 \\
\hline Frullania ericoides (Nees) Mont. & 1 & 1 & $\mathrm{BS}$ & 16.50 & 0.001 \\
\hline Frullania sp. & 0 & 1 & BS & 13.70 & 0.000 \\
\hline Frullania sp 1 & 1 & 0 & BS & 12.50 & 0.001 \\
\hline Frullania sp 2 & 0 & 1 & BP & 3.80 & 0.239 \\
\hline Frullania sp 3 & 0 & 1 & BS & 2.50 & 0.489 \\
\hline Lejeunea pterigonia (Lehm. \& Lindenb.) Mont. & 1 & 0 & BP & 11.20 & 0.004 \\
\hline Lejeunea sp. & 1 & 1 & $\mathrm{BP}$ & 16.30 & 0.377 \\
\hline Lejeunea sp 1 & 1 & 0 & BP & 2.50 & 0.490 \\
\hline Lejeunea sp 2 & 1 & 1 & $\mathrm{BP}$ & 4.10 & 0.388 \\
\hline Lejeunea sp 3 & 1 & 1 & BS & 24.20 & 0.000 \\
\hline Lejeunea $\mathrm{sp} 4$ & 1 & 1 & BS & 8.00 & 0.165 \\
\hline Lejeunea sp 5 & 0 & 1 & BS & 7.50 & 0.027 \\
\hline Lejeunea sp 6 & 0 & 1 & BS & 8.80 & 0.017 \\
\hline Lophocolea cf. aberrans Lindenb. \& Gottsche & 1 & 1 & BP & 1.20 & 1.000 \\
\hline Lophocolea sp. & 1 & 0 & $\mathrm{BP}$ & 10.80 & 0.049 \\
\hline Metzgeria ciliata Raddi. & 1 & 1 & BS & 11.20 & 0.120 \\
\hline Metzgeria furcata (L.) Corda & 1 & 1 & $\mathrm{BS}$ & 33.10 & 0.000 \\
\hline Metzgeria mexicana Steph. & 1 & 0 & BP & 3.80 & 0.244 \\
\hline Metzgeria sp. & 1 & 0 & $\mathrm{BP}$ & 5.00 & 0.130 \\
\hline Metzgeria sp1 & 0 & 1 & BS & 5.00 & 0.125 \\
\hline Plagiochila diversifolia Lindenb. \& Gottsche & 1 & 0 & $\mathrm{BP}$ & 41.20 & 0.000 \\
\hline Plagiochila laetevirens Lindenb. & 1 & 1 & BP & 13.90 & 0.027 \\
\hline Plagiochila raddiana Lindenb. & 0 & 1 & BS & 6.20 & 0.056 \\
\hline Plagiochila rutilans Lindenb. & 1 & 0 & $\mathrm{BP}$ & 1.20 & 1.000 \\
\hline Plagiochila simplex (Sw.) Lindenb & 1 & 1 & BP & 44.40 & 0.000 \\
\hline Plagiochila sp. & 0 & 1 & BS & 11.20 & 0.003 \\
\hline Plagiochila sp 1 & 0 & 1 & BS & 1.20 & 1.000 \\
\hline Plagiochila sp 2 & 0 & 1 & $\mathrm{BS}$ & 2.50 & 0.494 \\
\hline Plagiochila sp 3 & 0 & 1 & BS & 2.50 & 0.494 \\
\hline Plagiochila sp 4 & 0 & 1 & BS & 1.20 & 1.000 \\
\hline Porella complanata (Steph.) Swails & 1 & 0 & BP & 2.50 & 0.485 \\
\hline Porella crispata (Hook.) Trevis. & 1 & 1 & $\mathrm{BP}$ & 12.80 & 0.003 \\
\hline Radula cf. saccatiloba Stephani & 1 & 0 & BP & 2.50 & 0.483 \\
\hline Radula tectiloba Stephani & 1 & 0 & BP & 22.50 & 0.000 \\
\hline Radula voluta Taylor & 1 & 0 & $\mathrm{BP}$ & 2.50 & 0.508 \\
\hline
\end{tabular}

